\title{
La pédagogie universitaire en Belgique francophone : un processus qui s'inscrit dans la durée
}

University teaching in French-speaking Belgium: A process with a long view La pedagogía universitaria en Bélgica francófona: un proceso que se inscribe en el tiempo largo

Marcel Lebrun et Jean-Marie De Ketele

\section{OpenEdition}

Journals

Édition électronique

URL : https://journals.openedition.org/ries/8201

DOI : 10.4000/ries.8201

ISSN : 2261-4265

Éditeur

France Education international

Édition imprimée

Date de publication : 1 avril 2019

Pagination : 71-82

ISBN : 978-2-85420-623-4

ISSN : $1254-4590$

Référence électronique

Marcel Lebrun et Jean-Marie De Ketele, « La pédagogie universitaire en Belgique francophone : un processus qui s'inscrit dans la durée », Revue internationale d'éducation de Sèvres [En ligne], 80 | avril 2019, mis en ligne le 01 avril 2021, consulté le 24 juin 2021. URL : http://journals.openedition.org/ries/ 8201 ; DOI : https://doi.org/10.4000/ries.8201 


\title{
La pédagogie universitaire en Belgique francophone : un processus qui s'inscrit dans la durée
}

\author{
Marcel Lebrun \\ Jean-Marie De Ketele \\ Université catholique de Louvain
}

Comme dans d'autres pays, le développement de la pédagogie universitaire en Belgique s'inscrit dans un temps long et passe par diverses phases, dont le décollage et la durée varient d'une université à l'autre. Dans un premier temps, nous décrirons de manière historique, depuis les années 1970 jusqu'à aujourd'hui, l'évolution de la pédagogie universitaire dans notre institution, l'Université catholique de Louvain (UCLouvain). Ensuite, nous sélectionnerons et détaillerons quelques points essentiels de ce développement, en élargissant notre analyse à d'autres institutions et à différents réseaux.

\section{LA PÉDAgOGIE À L'UCLOUVAIN : APPROCHE HISTORIQUE}

\section{Une phase d'émergence}

En Belgique francophone, UCLouvain a été pionnière dans le développement de la pédagogie universitaire. Elle le doit à un vice-recteur à l'enseignement, Jean Demal, qui, dans les années 1970, a pris l'initiative de demander aux enseignants-chercheurs de se faire connaître, s'ils estimaient mettre en œuvre des « innovations » dans leur enseignement, c'est-à-dire des dispositifs et des pratiques différentes de celles que l'on observe habituellement. À l'étonnement général, plus de trois cents retours ont été reçus et ont fait l'objet d'une analyse par la conseillère du vice-recteur et un chercheur en éducation. Les plus originales, et tout particulièrement celles qui avaient fait l'objet d'une évaluation auprès des étudiants, ont été diffusées à l'ensemble de la communauté universitaire à travers le «feuillet vert». Celui-ci était une feuille verte A3, pliée en deux, décrivant plusieurs innovations menées dans l'institution et invitant les enseignants intéressés à prendre contact avec les auteurs concernés ou à solliciter une demi-journée de «formation » sur l'innovation en question.

De cette initiative est née une formule de formation qui a fait ses preuves et dont les étapes sont les suivantes :

- les auteurs de trois innovations (les «témoins ») sont sollicités pour effectuer un témoignage auprès de leurs collègues ; 
- une présentation de vingt à trente minutes est préparée par le témoin avec l'aide d'un expert en éducation, car l'expérience a montré que les auteurs d'une innovation sont rarement capables de la communiquer dans toute sa richesse aux participants ; souvent, ils oublient de mentionner certains aspects du processus d'innovation (comme le problème de départ et les difficultés rencontrées et à résoudre) ou considèrent comme évidents certains aspects, qui ne le sont pas pour les participants ;

- le déroulement de la séance se déroule en plusieurs étapes : une présentation des trois témoins par un animateur, les trois exposés des témoins, une discussion avec les participants, une discussion libre autour d'un verre de l'amitié, induisant très souvent de nouvelles relations entre collègues ;

- après la réunion, les témoins sont souvent amenés à jouer le rôle de coach auprès des enseignants qui tentent à leur tour d'innover dans leur enseignement.

Petit à petit, la pédagogie universitaire percolait dans la communauté enseignante. Les autorités, notamment à travers la commission de l'enseignement, ont valorisé les innovateurs et encouragé les collègues à faire de même, notamment en mettant à leur disposition des ressources, telles que l'expertise du personnel du Centre audiovisuel, des financements ou du personnel complémentaire pour les phases de conception. Elles ont également encouragé les enseignants à participer aux travaux de l'Association internationale de pédagogie universitaire (AIPU), créée en 1980 par Jean Demal. Ceci a incité de nombreux enseignants à mener des travaux de recherche collaborative, notamment sur les facteurs de réussite et d'échec des étudiants à la transition entre l'enseignement secondaire et supérieur ou sur les dispositifs pédagogiques innovants et leurs effets. L'association allait mettre en contact les enseignants belges avec des collègues d'autres pays, notamment du Canada, qui avait déjà, à l'époque, une expertise reconnue. C'est ainsi que les responsables louvanistes de la formation des ingénieurs, pionniers dans le domaine de la pédagogie universitaire, allèrent visiter l'approche par problèmes et par projets développée par la faculté de médecine de Sherbrooke, puis celle développée par Maastricht, toujours en médecine, et enfin celle mise en œuvre à l'École polytechnique de Copenhague. "Coachée » par ces institutions, la faculté des sciences appliquées (devenue l'École polytechnique) allait devenir à son tour coach pour d'autres institutions francophones. La pédagogie universitaire était en marche, une phase d'institutionnalisation pouvait commencer.

\section{L'institutionnalisation ${ }^{1}$}

L'institutionnalisation s'est faite progressivement en termes d'accompagnement pédagogique des enseignants, domaine dans lequel UCLouvain a été pionnière, en Europe occidentale en tout cas, en proposant à ces derniers un ensemble de mesures, de ressources, d'environnements dans lesquels ils allaient pouvoir apprendre, se développer et innover dans leurs pratiques. Avec un leadership pédagogique explicite, important et incontournable des autorités de l'Université, en interaction étroite avec une « commission de pédagogie » en toile de fonds, cet environnement favorable au développement pédagogique s'est constitué, selon nous, et a minima, à partir de quatre composants historiquement marqués mais qui, au final, interferent positivement.

1. Les aspects ci-dessous sont repris du document suivant : Louvain Learning Lab (2016). Carnet de l'enseignant, Voyages en pédagogie universitaire. [http://bit.ly/Carnet-LLL] 


\section{L'évaluation des enseignements}

Tout a commencé dès les années 1980, avec l'instauration de l'EEEQ (Évaluation des enseignements par les étudiants au moyen de questionnaires), dans le but de réguler la qualité des enseignements (et de documenter les décisions d'attribution de cours). Ces évaluations étaient alors classées dans un dossier relativement confidentiel appelé "dossier d'appréciations pédagogiques » (DAP). Un saut dans le temps nous conduit en 1992, avec une large étude consacrée à la réussite dans les premières années universitaires. Parmi les conclusions, nous lisons que la formation des enseignants à des formes plus interactives d'enseignement (centrées davantage sur l'apprenant) et l'évaluation de ces dernières constitueront un facteur important de réussite des actions pédagogiques entreprises. On y parle déjà de valorisation des enseignements (en particulier au premier cycle), mais sans plus.

\section{La formation des enseignants}

L'idée de la nécessaire formation des enseignants était en route et allait conduire à la création de l'IPM (Institut de pédagogie universitaire et des multimédias) en 1995. Dès décembre 1995, sept groupes de travail d'une quinzaine de personnes émanant des différents horizons de l'université (enseignants, étudiants, services...) produisent un dossier initiateur de nombreuses réformes ultérieures et intitulé « La pédagogie à l'Université ».

\section{Le soutien et le financement des initiatives pédagogiques}

En 1997, différents fonds épars souvent orientés « outils » (comme le fonds pour l'équipement, l'audiovisuel, le fonds multimédia...) sont fédérés dans le Fonds de développement pédagogique (FDP), marquant encore une fois cette volonté de considérer l'enseignement comme un moyen pour favoriser des apprentissages de qualité et d'investir dans ce domaine. Former les enseignants, évaluer leurs enseignements, les soutenir dans leurs intentions et actions pédagogiques, toute cette mouvance va conduire l'IPM à développer une approche permettant de répondre aux demandes et d'effectuer un accompagnement plus personnalisé des enseignants et surtout des équipes vis-à-vis de ce qui deviendra, vers 2010, l'approche programme.

\section{La valorisation des enseignants}

À la rentrée académique 2004-2005, un nouveau pas est franchi avec l'avènement d'un prorectorat à l'enseignement et à la formation, et avec l'explicitation d'une priorité fédératrice : "L'étudiant au centre de sa formation ; la formation centrée sur l'apprentissage ». Aussi, on y trace les prémisses du SoTL (Scholarship of Teaching and Learning $)^{2}$ même si celui-ci est alors encore peu explicite : l'enseignant-chercheur est invité à considérer son enseignement comme un objet de recherche, un objet

2. NdIR : ce concept anglo-saxon, difficile à traduire en français, désigne l'expertise dans l'enseignement. $(N d I R)$ Voir : N. Rege Colet, L. McAlpine, J. Fanghanel et C. Weston, « Le concept de Scholarship of Teaching and Learning ", Recherche et formation [En ligne], 67, 2011. DOI : 10.4000/rechercheformation.1412 
élaboré sur la base d'une expertise scientifique et fondé sur des preuves, à s'informer sur les pratiques pédagogiques et leurs finalités, à les expérimenter, à les évaluer, à les discuter avec des pairs et à les soumettre ainsi à la critique constructive et fertile en évolutions (la formation se muant en communauté de pratiques), et enfin à les publier à l'instar de ce qui se fait dans la recherche. La valorisation de la fonction enseignante à l'Université s'est alors traduite, de manière naturelle, par la mise en place du " dossier de valorisation pédagogique » (DVP) dès lors demandé pour les nominations et promotions dans la carrière.

On comprendra que, prenant appui sur ces nouvelles dimensions, l'IPM allait endosser de nouvelles fonctions, de nouvelles responsabilités. Elles sont traduites dans sa nouvelle appellation entrée en vigueur en 2015 de Louvain Learning Lab (LLL). Sans toutefois négliger les formations "générales " organisées depuis le tout début, l'accompagnement pédagogique des projets individuels et d'équipe, etc., le LLL propose désormais aux enseignants encore davantage d'occasions d'apprendre ensemble, dans un esprit d'innovation, de recherche de valeur ajoutée et de valorisation des efforts pédagogiques entrepris.

\section{La pratique ancrée : le développement du Learning Lab}

Les technologies ont toujours entretenu un lien étroit avec la pédagogie, que ce soit en tant que ressources pour les enseignants et les apprenants ou en tant qu'outils porteurs d'activités pédagogiques ou d'interactivités. Dans la foulée des centres audiovisuels qui préexistaient au Québec et en Europe (souvent orientés vers la production de ressources), et suite à différents rapports de la commission de pédagogie d'UCLouvain, fut créé en 1995 " un service interfacultaire de formation des enseignants ", déjà mentionné plus haut : l'institut de pédagogie universitaire et des multimédias ou IPM. Cette appellation marque la volonté de mixer le potentiel pressenti des technologies avec la nécessité de faire évoluer la pédagogie, voire d'assujettir l'approche technique au projet pédagogique. Il s'agit en fait d'intégrer de manière cohérente les outils dans l'alignement pédagogique (constructive alignment) de Biggs (2004), relatif aux objectifs, aux méthodes et aux évaluations ${ }^{3}$. C'est ainsi que dès 2000, au départ des besoins et des expériences d'enseignants, une plateforme générique mais adaptable fut développée afin de soutenir des projets pédagogiques à valeur pédagogique ajoutée : Claroline, l'un des premiers Learning Management Systems (LMS) développé en open source fut gratifié d'un prix Unesco $(2007)^{4}$.

À l'heure actuelle, les technologies sont devenues "le numérique ": les ressources éducatives libres (Open Educational Resources: OER), les nouveaux espaces

3. NdIR : John Biggs est le premier a avoir énoncé le concept d'alignement pédagogique : que faire acquérir aux étudiants, quelle approche pédagogique, quelles modalités d'évaluation?

4. Ce prix UNESCO pour l'utilisation des technologies de l'information et de la communication dans l'éducation a été décerné en 2007 à deux projets (Claroline et Curriki) choisis parmi 68 projets présentés par 51 pays. [http:// bit.ly/Claroline-2007] 
d'apprentissage (Learning Spaces), les méthodes de créativité, etc., ouvrent le champ de la formation à de nouveaux possibles. Certes, l'outil reste un outil mais le numérique transforme radicalement les activités humaines, du champ socioprofessionnel à la vie politique, économique, les activités quotidiennes, de loisirs... Il change aussi nos façons d'apprendre et d'enseigner, en modifiant les compétences nécessaires et en devenant in fine bien plus qu'un outil. C'est ainsi que le Louvain Learning Lab (nom récent de l'IPM) se veut un laboratoire dans lequel sont développées et expérimentées « scientifiquement» de nouvelles approches pédagogiques telles que les MOOCs (Massive Open Online Course) ou les classes inversées.

\section{LES PILIERS ACTUELS DE LA PÉDAGOGIE UNIVERSITAIRE}

Avec des variantes et des rythmes différents, les trois phases du développement de la pédagogie universitaire à Louvain (émergence, institutionnalisation, pratique ancrée) caractérisent aussi celui des autres universités francophones de Belgique, comme l'Université de Liège, l'Université libre de Bruxelles, l'Université Saint Louis, l'Université de Namur et l'Université de Mons. Actuellement, on peut dire que la pédagogie universitaire dans toutes ces universités tend à reposer sur les piliers suivants : des dispositifs d'aide aux étudiants ; l'évaluation des enseignements par les étudiants (EEE) et la prise en compte progressive de la fonction pédagogique dans les nominations et promotions ; la professionnalisation du métier d'enseignant tant dans les universités que dans les Hautes écoles ${ }^{5}$; la professionnalisation également du personnel des centres de ressources pédagogiques et technologiques au service de l'enseignement ; la valorisation de la recherche en pédagogie universitaire ; la montée en puissance des dispositifs d'évaluation de la qualité des institutions d'enseignement supérieur.

\section{DES DISPOSITIFS D'AIDE AUX ÉTUDIANTS À QUESTIONNER}

Face à la massification et à l'hétérogénéisation de l'enseignement supérieur entraînant des risques d'échec de plus en plus traumatisants pour les étudiants et leurs familles, largement répercutés par les médias, les universités belges ont réagi en proposant des dispositifs d'aide et de soutien. L'éventail des expérimentations au fil du temps est très large, au point que des chercheurs ont tenté d'en dresser des typologies. Plusieurs critères à croiser sont nécessaires pour en montrer leur variété. Nous présentons ci-dessous (tableau 1) ceux qui nous paraissent prioritaires.

5. En Belgique, l'enseignement supérieur est composé des universités et des hautes écoles, spécialisées dans la formation de certains métiers. Chaque haute école est désormais attachée à une université, tout en gardant une certaine indépendance. 


\section{Tableau 1}

\section{Critères prioritaires des dispositifs d'aide aux étudiants en Belgique}

\begin{tabular}{|c|c|}
\hline $\begin{array}{l}\text { Critère 1. Population étudiante } \\
\text { concernée par le dispositif }\end{array}$ & $\begin{array}{l}\text { - population en décrochage } \\
\text { - population en difficulté } \\
\text { - population à risque } \\
\text { - toute la population }\end{array}$ \\
\hline Critère 2. Degré d'obligation & $\begin{array}{l}\text { - participation totalement libre } \\
\text { - participation conseillée } \\
\text { - participation obligatoire }\end{array}$ \\
\hline $\begin{array}{l}\text { Critère } 3 \text {. Personnel chargé de l'aide } \\
\text { ou du soutien }\end{array}$ & $\begin{array}{l}\text { - pairs (étudiants des années supérieures ou étudiants } \\
\text { brillants de l'année) } \\
\text { - enseignants du secondaire } \\
\text { - enseignants chercheurs } \\
\text { - professionnels de l'accompagnement }\end{array}$ \\
\hline $\begin{array}{l}\text { Critère } 4 \text {. Objet de l'aide } \\
\text { ou du soutien }\end{array}$ & $\begin{array}{l}\text { - contenu disciplinaire } \\
\text { - méthodologie du travail universitaire } \\
\text { - intégration sociale et institutionnelle } \\
\text { - projet personnel }\end{array}$ \\
\hline $\begin{array}{l}\text { Critère } 5 \text {. Moment du soutien } \\
\text { ou de l'aide }\end{array}$ & $\begin{array}{l}\text { - avant l'entrée à l'université } \\
\text { - à l'entrée (dans les premières semaines) } \\
\text { - tout au long de l'année } \\
\text { - avant une session d'examens } \\
\text { - après la session d'examens de janvier } \\
\text { - après la première année }\end{array}$ \\
\hline
\end{tabular}

Le croisement des modalités de ces cinq critères aboutit à de nombreux dispositifs différents, dont beaucoup ont été expérimentés pour tenter de répondre à la double question de leur efficacité (effets sur le parcours de l'étudiant) et de leur pertinence (adéquation aux besoins des étudiants et aux moments opportuns). Conscientes de ces enjeux mais également soucieuses des coûts de tels dispositifs, les autorités académiques ont encouragé les chercheurs à mener des recherches évaluatives à ce sujet ${ }^{7}$. Même s'il reste de nombreuses questions en suspens, on peut dégager quelques résultats intéressants : dans les dispositifs où la participation est libre, ce sont les étudiants les plus motivés et qui n'ont pas nécessairement le plus besoin du dispositif d'aide qui en profitent le plus; les dispositifs centrés sur les contenus disciplinaires ont plus d'impact sur les résultats académiques que ceux centrés sur les aspects méthodologiques, tout particulièrement dans les filières scientifiques ; les dispositifs ont souvent un impact positif sur l'intégration sociale et institutionnelle des étudiants, mais non nécessairement sur leurs résultats académiques, du moins à court terme.

6. En Belgique, l'enseignement supérieur universitaire est semestrialisé. Il existe donc une session d'examens en janvier.

7. La recherche dont la référence est mentionnée ci-dessous est intéressante, non seulement parce qu'elle compare l'efficacité de deux types de dispositifs importants mais aussi parce qu'elle dresse une revue critique internationale importante des travaux antérieurement menés sur les dispositifs d'aide : Vertongen G., Nils F., Galdiolo S. et al. (2015) : "Test de l'efficacité de deux dispositifs d'aide à la réussite en $1^{\text {re }}$ année d'université : remédiations précoces et blocus dirigés ", Louvain-la-Neuve : Cahiers du Girsef, n ${ }^{\circ} 103,26$ p. Ces résultats ont été aussi démontrés en France dans les recherches de C. Michaut (2003) : " L'efficacité des dispositifs d'aide aux étudiants dans les universités ", Recherche et Formation, 43, p. 101-113. 


\section{De L’ÉVALUATion \\ DES ENSEIGNEMENTS \\ PAR LES ÉTUDIANTS AU DOSSIER \\ DE VALORISATION PÉDAGOGIQUE}

S'inspirant des pratiques du monde anglo-saxon, les facultés belges chargées de la formation des ingénieurs ont compris les premières (dès les années 1970, pour certaines d'entre elles) l'intérêt de faire évaluer les enseignements par les étudiants (EEE). Cette pratique est entrée désormais dans les mœurs de tous les départements, car à l'usage, les enseignants chercheurs se sont aperçus que la plupart de leurs craintes étaient infondées. Au fil du temps, s'inspirant des nombreuses recherches sur l'EEE, les institutions ont mis en place des dispositifs rôdés : centration sur ce que les étudiants étaient les mieux à même d'évaluer ; création de banques d'items adaptés aux différentes formes d'enseignement ; traitements automatisés sur la base de procédures mises au point avec des spécialistes; communication des résultats à l'enseignant concerné, qui peut ainsi se situer par rapport aux résultats d'un collectif d'enseignants sur les différents critères pris en considération ; possibilité pour celui-ci de solliciter un soutien auprès d'une instance de conseil ; adjonction à l'évaluation proprement dite de l'avis de l'enseignant, en fonction de son contexte et des mesures éventuellement prises ; prise en considération d'un ensemble d'EEE (et non d'une seule) ainsi que d'autres prises d'informations en provenance d'autres acteurs (par exemple : analyse des supports de cours par une commission des programmes, audit externe d'un programme d'enseignement, etc.). Ainsi, les institutions ont trouvé au fil du temps le moyen de concilier la fonction formative (développement professionnel) et certificative (évolution dans la carrière) de l'évaluation.

Le dispositif actuel, probablement le plus abouti, est sans doute le « dossier de valorisation pédagogique " $(\mathrm{DVP})^{8}$, désormais exigé pour les nominations et les promotions de carrière, mais surtout conçu pour être un outil de développement professionnel, dans un contexte où existent des tensions entre les missions de recherche, d'enseignement et de services. Le DVP repose sur les travaux du courant anglo-saxon du "Scholarship of Teaching and Learning 》 (SoTL), qui distingue trois niveaux d'expertise dans le développement professionnel de l'enseignant universitaire : (1) il développe dans son enseignement des compétences réelles ; (2) il échange ses expériences avec d'autres enseignants et développe avec eux certains projets ; (3) il contribue à produire des connaissances dans le champ de l'enseignement et de l'apprentissage. Avec l'aide d'un conseiller pédagogique, s'il le souhaite, l'enseignant va constituer un DVP qui contiendra les éléments représentatifs de son activité sur le plan pédagogique, éléments qui seront analysés par une commission à l'aide d'une grille basée sur les trois niveaux d'expertise mentionnés ci-dessus. Ainsi, les résultats de plusieurs EEE seront des traces relatives au niveau 1 , tandis que des communications ou des publications dans le cadre d'une association de pédagogie universitaire relèveront du niveau 3. À côté du CV scientifique, le DVP devient un élément important dans la carrière de l'enseignant chercheur.

8. Le DVP a fait l'objet d'une décision du conseil académique de l’UCL en 2000, mais n'a été vraiment mis en pratique qu'aux environs de $\mathbf{2 0 0 7}$, le temps que les responsables et commissions facultaires ou départementales se l'approprient. 


\section{VERS UNE PROFESSIONNALISATION DU MÉTIER D'ENSEIGNANT UNIVERSITAIRE}

Les actions menées au fil du temps (dispositifs d'aide aux étudiants, EEE, DVP) ont instillé une prise de conscience de la complexité de l'acte d'enseignement et du processus d'apprentissage et de la nécessité bénéfique de se développer sur le plan professionnel. La fonction d'enseignant dans l'enseignement supérieur devait donc se professionnaliser. Au départ, il s'agissait de participer aux offres de formation offertes par l'institution et de saisir les opportunités offertes pour s'engager dans des projets d'amélioration de son enseignement ou dans des projets innovants. Avec des dispositifs comme le DVP et les offres de «Master en pédagogie universitaire et de l'enseignement supérieur » dans toutes les universités belges, un pas supplémentaire est franchi vers plus de professionnalisation du métier d'enseignant dans l'enseignement supérieur. Les doctorants et les enseignants-chercheurs nouvellement nommés sont encouragés à suivre ces masters complémentaires centrés sur la pratique professionnelle, sur la réalisation de projets de formation, sur l'échange et l'analyse de pratiques diverses. Les bénéficiaires peuvent ainsi disposer d'apports utiles pour répondre aux exigences institutionnelles de début de carrière ; au-delà de la formation, ils poursuivent souvent les échanges avec les collègues de leur promotion ou les formateurs ; ainsi se crée progressivement une communauté d'apprentissage au sein de l'institution ou avec d'autres institutions.

\section{VERS UNE PROFESSIONNALISATION DES CENTRES DE RESSOURCES ET DE SOUTIEN}

De manière générale, les « centres de pédagogie universitaire » contribuent à la formation et à l'accompagnement pédagogiques des enseignants. Dans une société en mutation où les savoirs et les savoir-faire se démultiplient et se diffusent rapidement, les enseignants, comme dans la plupart des métiers, continuent à apprendre tout au long de la vie. Ainsi, dans un même mouvement, les apprentissages des étudiants et ceux des enseignants préparent notre société en devenir. Même si leur métier est récent et surtout parce qu'il est récent, les conseillers ou ingénieurs pédagogiques, à la racine de ce mouvement de transformation de la pédagogie, se doivent aussi de veiller à leur propre formation continue et de se doter des moyens de continuer à apprendre. Comme les enseignants, ils s'inscrivent dans une démarche SoTL en mettant en place des pratiques d'accompagnement innovantes, en analysant ces dernières, en les soumettant à la critique de leurs pairs, en les diffusant de manière nationale et internationale. Nous en présentons ci-dessous trois exemples.

\section{Le BSQF (Belgique, Suisse, Québec, France)}

À l'issue du congrès de l'Association internationale de pédagogie universitaire (AIPU) d'avril 2000 à Nanterre, quelques acteurs de centres de formation des 
enseignants d'alors (citons A. Laloux et M. Lebrun de l'IPM à Louvain-la-Neuve, R. Prégent et $\mathrm{H}$. Bernard du Bureau d'appui pédagogique à Montréal), faisant le constat du manque de communications relatives à l'accompagnement pédagogique des enseignants, décidèrent de la constitution d'un réseau de conseillers pédagogiques : le premier BSQ (initiales de Belgique, Suisse, Québec) tint ses rencontres en 2001 à Magog (Québec). Il s'agissait de présenter, critiquer, modéliser et valider des pratiques relatives au conseil pédagogique, en les alimentant de savoirs pédagogiques et en les faisant vivre au travers de méthodes pédagogiques innovantes. Vers 2010, la France s'ajoutait à ce réseau international francophone qui est devenu, depuis, le BSQF ; le dernier colloque s'est tenu en 2017 en Bretagne sur le thème de la transformation pédagogique.

\section{La collaboration Louvain-Laval}

Après cette approche «bottom-up », nous présentons une autre initiative de formation mutuelle de conseillers pédagogiques et d'acteurs institutionnels, cette fois dans le cadre d'une collaboration institutionnelle des universités de Louvain (Belgique) et de Laval (Québec). Au départ, il s'agissait principalement de rencontres entre acteurs institutionnels impliqués à différents niveaux dans la pédagogie universitaire et de conseillers pédagogiques ; les travaux étaient constitués de partages et d'analyses de pratiques sur différentes thématiques, comme l'évaluation des enseignements et des programmes, les approches d'élaboration de curricula par compétences et par programmes de formation (la problématique des learning outcomes, par exemple). À l'heure actuelle, ces échanges ont abouti à la mise en place d'universités d'été, avec la participation d'enseignants particulièrement actifs dans les thématiques choisies. En 2018, la thématique était celle de la créativité, ses enjeux, les compétences et démarches mises en place et en 2019 il fut question des espaces d'apprentissage.

\section{Le réseau des Learning Labs}

Depuis quelques années, la problématique des espaces d'enseignement et d'apprentissage est apparue dans les réflexions sur les compétences à développer chez les étudiants, sur les méthodes actives et interactives, sur les enjeux du numérique aux échelles sociétale et éducative. En effet, par un mécanisme d'affordance ${ }^{9}$, les outils numériques et les espaces dans lesquels se tiennent les formations peuvent soutenir et même entraîner de nouveaux usages pédagogiques plus qualitatifs en termes d'apprentissages et de compétences déployées. Après des expériences pilotes (mentionnons l'École centrale de Lyon et EMLyon dès 2010, par exemple), un réseau international de Learning Labs s'est constitué, en réunissant des institutions disposant d'espaces innovants entièrement dédiés aux nouvelles formes d'apprentissage et utilisant les possibilités offertes par les technologies numériques. La charte de ce réseau, en accord avec le principe de développement pédagogique soutenu par la recherche expérimentale, repose sur six points :

- favoriser l'innovation pédagogique en testant et en développant différents modes d'apprentissage ;

9. Capacité à, potentialité, en anglais. (NdIR) 
- disposer d'un espace innovant, au sein d'un établissement d'enseignement, d'un organisme de formation ou d'une entreprise, permettant d'accueillir des formations, des rencontres, des séminaires, des productions, des expérimentations, des recherches, de l'observation ;

- disposer d'un ensemble d'équipements numériques dédiés à l'appui pédagogique ;

- s'appuyer sur un dispositif de recherche sur l'innovation pédagogique ;

- soutenir l'acquisition des compétences, des cultures et des humanités numériques ;

- être un lieu ouvert accueillant des publics et des événements variés.

\section{LA RECHERCHE EN PÉDAGOGIE UNIVERSITAIRE AU CEUR DU DÉVELOPPEMENT PROFESSIONNEL COLLECTIF ET PERSONNEL}

Au fil des ans, la recherche en pédagogie universitaire a pris une ampleur et une importance considérable en Belgique francophone. De nombreuses études empiriques ont été menées sur des thèmes aussi divers que les facteurs de réussite et d'échec, l'évaluation des enseignements, les effets des dispositifs d'enseignement et d'apprentissage, les effets de l'introduction du numérique sur les conceptions pédagogiques, etc. Ces recherches ont été coordonnées le plus souvent par des chercheurs experts du domaine, mais ont impliqué très fréquemment des collaborations avec les responsables et les enseignants des facultés et départements intéressés par l'objet de la recherche. Dans chaque université, on relève au moins une équipe de recherche dont les travaux ont acquis une grande audience internationale. Citons, à titre d'exemples sans être exhaustifs, les institutions suivantes : le LabSET (Laboratoire de soutien aux synergies éducation-technologie) de l'Université de Liège ; la cellule PRAC-TICE (Pédagogie, recherche-action \& TICE) de l'Université libre de Bruxelles ; la CFPU (Cellule facultaire de pédagogie universitaire) de l'Université de Mons ; le SPU (Service de pédagogie universitaire) de l'Université de Namur ; les trois institutions d'UCLouvain travaillant en collaboration, à savoir la Chaire Unesco de pédagogie universitaire, le LLL (Louvain Learning Lab) et le GIRSEF (Groupe interfacultaire de recherche sur la socialisation, la formation et l'éducation). Ces institutions de recherche jouent un rôle important dans le développement de la pédagogie universitaire au sein de leur institution, mais également au niveau international, en étant des membres actifs de l'AIPU (et d'autres associations comme l'ADMEE ${ }^{10}$ ou le $\mathrm{REF}^{11}$...) et en menant des recherches conjointes avec des chercheurs étrangers. Les références de quelques-unes d'entre elles sont mentionnées en fin d'article. 


\section{L'ÉVALUATION DE LA QUALITÉ DES INSTITUTIONS D'ENSEIGNEMENT SUPÉRIEUR}

Le processus de Bologne a contribué à créer un espace européen de l'enseignement supérieur qui réunit aujourd'hui 47 pays. L'organisation des études en trois cycles et la mobilité Erasmus (plusieurs millions d'étudiants) en sont les principaux fleurons. Avec la multiplication des échanges s'est posée la question de l'équivalence de la qualité de la formation donnée. De nombreuses tentatives (définition de standards de qualité, développement de guidelines, inventaire de bonnes pratiques) ont été lancées pour assurer une plus grande homogénéité entre les formations assurées par les différents partenaires européens. Au niveau européen, on a vu naître l'European Association for Quality Assurance in Higher Education (ENQA) ; au niveau des pays francophones, le réseau FrAQ-Sup (Réseau francophone des agences qualité pour l'enseignement supérieur) ; au niveau de chaque pays, une instance d'évaluation de la qualité, dont l'AEQES (Agence d'évaluation de la qualité de l'enseignement supérieur) pour la Belgique francophone ${ }^{12}$.

De telles institutions ont amené les institutions belges d'enseignement supérieur à se poser la question fondamentale « Qu'est-ce qu'un enseignement supérieur de qualité ? » et à se positionner par rapport à différentes conceptions de la qualité : excellence réservée à quelques-uns ? absence de défaut ? atteinte de l'objectif fixé par l'institution ? rapport coûts/bénéfices ? processus de transformation par les acteurs eux-mêmes et pour les acteurs ? Face à des institutions d'enseignement supérieur très diversifiées (en termes de missions et contextes), la tâche de l'AEQES est ardue, car il s'agit de prendre en compte les exigences européennes (ENQA), tout en ne négligeant pas les enjeux spécifiques des institutions belges, dont certaines visent un bon classement dans les classements internationaux et d'autres s'inscrivent dans une niche particulière (comme la réponse à des problèmes de développement régional ou aux besoins d'un champ professionnel).

Pour faire face aux tensions qu'une telle situation engendre, l'AEQES tente de concilier à la fois une approche bottom-up et une approche top-down. La première conduit chaque institution, avec ses spécificités, à adopter une démarche qualité dans laquelle les acteurs sont invités à répondre aux questions suivantes :

1. Que sommes-nous en train de réaliser?

2. Pourquoi le faisons-nous?

3. Comment le faisons-nous?

4. Est-ce que notre manière de la faire est la meilleure ? Peut-on l'optimiser?

5. Est-ce que cela fonctionne?

6. Peut-on s'améliorer?

Cette démarche qualité s'inscrit dans une conception de la qualité comme processus de transformation s'inscrivant dans la durée et pouvant convenir à des institutions vivant des contextes différents et recherchant des finalités spécifiques. L'approche top-down est présente dans l'évaluation externe des institutions, telle qu'elle est pratiquée maintenant par la plupart des agences nationales : un comité interna-

12. Nous remercions notre collègue Philippe Parmentier, Président de l'AEQES, pour les informations fournies. 
tional d'experts externes (à la lumière des standards européens) jette un regard sur le rapport interne de l'institution (résultat de l'approche bottom-up ci-dessus), mène éventuellement des entretiens complémentaires et propose un plan de suivi.

Surtout si elles sont bien conduites, ces deux approches complémentaires induisent progressivement une culture de la qualité dans les institutions, répondant mieux à la fois à des standards généraux de l'espace européen (les piliers de la pédagogie universitaire présentés ci-dessus tendent à s'y retrouver) et à des critères opérationnels répondant aux besoins spécifiques de l'institution.

\section{RÉFÉRENCES BIBLIOGRAPHIQUES}

BIGGS J. (2014) : «Constructive alignment in university teaching », HERDSA Review of Higher Education, 1, p. 5-22. Hammondville (Australia) : HERDSA.

DE KETELE J.-M., HUGONNIER B., PARMENTIER P. et COSNEFROY L. (sous la direction de) (2016). Quelle excellence pour l'enseignement supérieur? Louvain-la-Neuve : De Boeck supérieur.

LEBRUN M. (2007) : « Quality towards an expected harmony: Pedagogy and technology speaking together about innovation ", AACE Journal, 15(2), p. 115-130, Chesapeake, VA: AACE.

LEBRUN M., PELTIER C., PERAYA D., BURTON R. et MARCUSO G. (2014) : « Un nouveau regard sur la typologie des dispositifs hybrides de formation ", Éducation \& Formation, e-301, p. 55-74.

POUMAY M., TARDIF J. et GEORGES F. (sous la direction de) (2017) : Organiser la formation à partir des compétences. Un pari gagnant pour l'apprentissage dans le supérieur, Louvain-la-Neuve : De Boeck supérieur.

RAUCENT B., VERZAT C. et VILLENEUVE L. (sous la direction de) (2010) : Accompagner des étudiants. Quels rôles pour l'enseignant? Quels dispositifs ? Quelles mises en cuvre?, Louvain-la-Neuve : De Boeck supérieur.

ROMAINVILLE M. et MICHAUT C. (sous la direction de) (2012) : Réussite, échec et abandon dans l'enseignement supérieur, Louvain-la-Neuve : De Boeck supérieur.

WARNIER P., WARNIER L., PARMENTIER P., LELOUP G., PETROLITO S. (2010) : « Et si on commençait par les résultats ? Élaboration d'une démarche de définition des acquis d'apprentissage d'un programme de formation universitaire ", Actes $d u 26^{e}$ congrès de l'AIPU, Rabat (Maroc). 